\title{
FOXP3-induced LINC00885 promotes the proliferation and invasion of cervical cancer cells
}

\author{
YAWEN LIU ${ }^{1,2}$, HAIYAN TU ${ }^{2}$, LINGLING ZHANG ${ }^{2}$, JIANPING XIONG ${ }^{3}$ and LING LI $^{2}$ \\ ${ }^{1}$ Medical College of Nanchang University; ${ }^{2}$ Department of Gynecological Oncology, Jiangxi Maternal and \\ Child Health Hospital; ${ }^{3}$ Department of Oncology, The First Affiliated Hospital of Nanchang University, \\ Nanchang, Jiangxi 330006, P.R. China
}

Received September 18, 2020; Accepted January 7, 2021

DOI: $10.3892 / \mathrm{mmr} .2021 .12097$

\begin{abstract}
Cervical cancer (CC) is the fourth most common type of cancer in women worldwide. LINC00885, a novel oncogenic type of long non-coding RNA, is upregulated in various types of cancer, but its expression in CC remains unknown. The aim of the present study was to investigate the expression of LINC00885 in CC, and its effect on the proliferation and invasion of $\mathrm{CC}$ cells in vitro. The expression levels of LINC00885 and forkhead box protein 3 (FOXP3) from The Cancer Genome Atlas (TCGA) were selected to analyze the differences between $\mathrm{CC}$ tissues and normal cervical tissues using bioinformatics analyses. Reverse transcription-quantitative (RT-q)PCR was used to detect the relative expression of LINC00885 in CC cell lines, and its expression was knocked down. Cell Counting Kit- 8 assays and EdU staining were used to detect the changes in cell proliferative capacity of cells. Transwell experiments were used to examine cell invasion. Dual-luciferase reporter and chromatin immunoprecipitation assays were performed to examine the interactions between LINC00885 and FOXP3. FOXP3 and epithelial-mesenchymal transition (EMT)-related proteins were detected using western blotting. The expression of LINC00885 and FOXP3 in CC tissues and CC cells was significantly higher compared with the normal cervical tissues and normal cervical epithelial cells. FOXP3 could specifically interact with the promoter of LINC00885 and activate LINC00885 transcription. Knockdown of LINC00885 and silencing of FOXP3 significantly inhibited proliferation,
\end{abstract}

Correspondence to: Dr Jianping Xiong, Department of Oncology, The First Affiliated Hospital of Nanchang University, 17 Yongwai Street, Nanchang, Jiangxi 330006, P.R. China

E-mail: xjpingd@126.com

Dr Ling Li, Department of Gynecological Oncology, Jiangxi Maternal and Child Health Hospital, 318 Bayi Road, Nanchang, Jiangxi 330006, P.R. China

E-mail: lilingjxnc@hot.com

Key words: cervical cancer, LINC00885, forkhead box protein 3, proliferation, invasion invasion and EMT of CC cells. Overexpression of LINC00885 reversed the inhibitory effects of FOXP3 knockdown on the proliferation and invasion of CC cells. Collectively, LINC00885 and FOXP3 may serve as biomarkers for the early diagnosis of $\mathrm{CC}$ and as a potential therapeutic target for reversing the malignant phenotype of $\mathrm{CC}$.

\section{Introduction}

Cervical cancer (CC) is the fourth most common type of cancer in women worldwide and the leading cause of cancer-related death in some of the poorest countries in the world (1). Recent global data estimated 527,624 new cases and 265,672 deaths due to CC during 2018 (2). The 5-year survival rate for metastatic CC is $16.5 \%$ compared with $91.5 \%$ for localized CC (3). CC is preventable via the human papillomavirus (HPV) vaccination and cervical screening with primary HPV testing followed by treatment of any detected precancerous lesions (4). Unfortunately, the majority of women in developing countries still do not have access to CC prevention programs, resulting in increased CC disease burden. To improve prevention of CC and cancer treatment outcomes, there is an urgent need to consider exploring an effective and simple means of diagnosing and treating $\mathrm{CC}$.

Long non-coding RNAs (lncRNAs) are a class of single-stranded RNAs, $>200$ nucleotides in length, which do not possess protein coding capacity. However, they do contribute to the modulation of tumor progression via regulation of biological processes through chromatin remodeling, and transcriptional and post-transcriptional processing $(5,6)$. Recent studies have reported that dysregulated expression of IncRNAs are likely pervasive in human cancer types, and can drive cancer development and progression of several types of cancer, such as colorectal, breast and gastric cancer (7-9). LINC00885, a novel oncogenic lncRNA type, was upregulated in breast cancer cell lines (10). LINC00885 may also serve roles in preventing the progression and metastasis of bladder cancer (11). However, the relationship between LINC00885 and $\mathrm{CC}$ has not yet been reported.

Forkhead box protein 3 (FOXP3) $^{+}$cells are generally considered to suppress antitumor immune responses in several types of cancer, such as gastrointestinal cancer and gallbladder carcinoma $(12,13)$. Immune dysregulation, 
polyendocrinopathy, enteropathy and X-linked syndrome are caused by different genetic defects in the FOXP3 gene (14). FOXP3 is a specific transcription factor that is expressed on the surface of regulatory $\mathrm{T}$ cells (Tregs), which serves important roles in the tumor microenvironment and participates in tumor cell growth, invasion and metastasis, in breast, gastric, thyroid and non-small cell lung cancer (15-18). FOXP3 overexpression leads to severe immunodeficiency and $\mathrm{FOXP}^{+}$Tregs are abundant in tumor infiltrates and peripheral blood from patients with cancer, where it is often associated with a poor prognosis (19). Importantly, FOXP3 is highly expressed in CC, and able to facilitate proliferation and invasiveness, resulting in the occurrence, development and metastasis of $\mathrm{CC}$, and FOXP3 serves an important role in lymph angiogenesis of CC $(20,21)$.

The present study aimed to examine the roles of LINC00885 in CC in vitro. The results showed that LINC00885 can interact with FOXP3 in CC cells. Moreover, the heterologous overexpression of LINC00885 in cultured CC cells promoted cell proliferation, invasion and EMT. Therefore, LINC00885 may be a potential therapeutic target for the treatment of CC.

\section{Materials and methods}

Expression of genes in cervical tumor samples. Expression levels of LINC00885 and FOXP3 in CC tumor samples in patients were examined using Gene Expression Profiling Interactive Analysis 2 (GEPIA2; gepia2 .cancer-pku.cn/\#index), using data obtained from The Cancer Genome Atlas (TCGA; cancer.gov/tcga) and the Genotype Tissue Expression (GTEx) project (22-24).

Cell lines and culture. The human CC cell lines (C-33A, $\mathrm{SiHa}, \mathrm{HeLa}$ and $\mathrm{CaSki}$ ) and normal cervical epithelial cell line (Ect1/E6E7) were purchased from The Cell Bank of Type Culture Collection of the Chinese Academy of Sciences, and maintained in RPMI-1640 medium (Thermo Fisher Scientific, Inc.) supplemented with 10\% FBS (Invitrogen; Thermo Fisher Scientific, Inc.) and $100 \mathrm{U} / \mathrm{ml}$ penicillin $/ 100 \mu \mathrm{g} / \mathrm{ml}$ streptomycin (Invitrogen; Thermo Fisher Scientific, Inc.) at $37^{\circ} \mathrm{C}$ with $5 \% \mathrm{CO}_{2}$. Cells in the logarithmic growth phase were harvested for further experiments.

Cell transfection. For optimal short hairpin (sh)RNA transfection efficiency, two shRNA sequences were designed to target the human LINC00885 gene and one shRNA sequence was designed to target the human FOXP3 gene. The LINC00885 shRNA-1 (sh-LINC00885\#1) and LINC00885 shRNA-2 (sh-LINC00885\#2), FOXP3 shRNA (sh-FOXP3) and control shRNA (sh-NC) were obtained from Shanghai GenePharma Co., Ltd. A LINC00885 overexpression plasmid, pcDNA3.1-LINC00885, was commercially constructed by Shanghai GenePharma Co., Ltd., and an empty pcDNA 3.1 vector $(\mathrm{NC})$ was used as the control. Cells $\left(2 \times 10^{5}\right.$ cells/well $)$ were added to 6 -well plates and transfected with $50 \mathrm{nmol} / \mathrm{l}$ sh-LINC00885\#1, sh-LINC00885\#2, sh-FOXP3, sh-NC, pcDNA3.1-LINC00885 or pcDNA3.1, or co-transfected with sh-FOXP3 and pcDNA3.1 or pcDNA3.1-LINC00885 using Lipofectamine ${ }^{\circledR} 2000$ (Invitrogen; Thermo Fisher scientific, Inc.) according to the manufacturer's protocol. At
$48 \mathrm{~h}$ post-transfection, cells were harvested for subsequent experiments.

Reverse transcription-quantitative (RT-q)PCR. Total RNA was isolated from CC cell lines using TR Izol ${ }^{\circledR}$ reagent (Invitrogen; Thermo Fisher Scientific, Inc.) according to the manufacturer's protocol. For LINC00885 expression analysis, the RNA was reverse transcribed into cDNA using a High Capacity cDNA Reverse Transcription kit (Thermo Fisher Scientific, Inc.) according to the manufacturer's instructions. Subsequently, qPCR was performed using the SYBR Premix Ex Taq ${ }^{\mathrm{TM}}$ II kit (Thermo Fisher Scientific, Inc.) using an ABI Prism 7700 sequence detector (Applied Biosystems; Thermo Fisher Scientific, Inc.). The sequences of the PCR primers used were: LINC00885 forward, 5'-CAGGGTTGGTGCTATGAATGAC-3' and reverse, 5'-GAAGATTGTCCATGTTGGCAGTAT-3'; and GAPDH forward, 5'-CCATCTTCCAGGAGCGAGAT-3' and reverse, 5'-TGCTGATGATCTTGAGGCTG-3'. The following thermocycling conditions were used for qPCR: Initial denaturation at $95^{\circ} \mathrm{C}$ for $10 \mathrm{~min}$; followed by 40 cycles of $94^{\circ} \mathrm{C}$ for $2 \mathrm{~min}, 60^{\circ} \mathrm{C}$ for $50 \mathrm{sec}$; a final extension at $60^{\circ} \mathrm{C}$ for $1 \mathrm{~min}$. All reactions were performed at least three times. GAPDH was used as the house-keeping gene. The formula $2^{-\Delta \Delta C q}$ was used to calculate relative gene expression (25).

Cell proliferation assay. A Cell Counting Kit-8 (CCK-8; Beijing Solarbio Science \& Technology Co., Ltd.) assay was used to analyze cell proliferation, according to the manufacturer's instructions. Briefly, transfected CaSki and HeLa cells $\left(1 \times 10^{4}\right.$ cells/well) were seeded in 96-well plates for $0,24,48$ or $72 \mathrm{~h}$, and subsequently $10 \mu \mathrm{l}$ CCK- 8 reagent was added to the medium and further incubated in the dark at $37^{\circ} \mathrm{C}$ for $2 \mathrm{~h}$. The absorbance of every well was measured at $450 \mathrm{~nm}$ using a microplate reader.

EdU staining assay. EdU staining was used to detect proliferation. Transfected CaSki and HeLa cells $\left(4 \times 10^{4}\right.$ cells/well) were plated into a $96-$ well plate and incubated with $20 \mu \mathrm{M} \mathrm{EdU} \mathrm{(Thermo} \mathrm{Fisher} \mathrm{Scientific,} \mathrm{Inc.)} \mathrm{for} 3 \mathrm{~h}$. Cells were analyzed under a confocal microscope (magnification, x200; Leica Microsystems GmbH). DNA (blue) was stained using DAPI for $10 \mathrm{~min}$ at room temperature. Green cells were the EdU/DAPI-positive cells.

Transwell assay. Transwell assays were used to measure the invasive ability of the transfected cells. The 24-well Transwell chambers $(8-\mu \mathrm{m}$ pores) pre-coated with Matrigel (BD Biosciences) overnight at $37^{\circ} \mathrm{C}$ were used to assess invasion. A total of $200 \mu 1$ media with transfected CaSki and HeLa cells $\left(5 \times 10^{4}\right.$ cells $\left./ \mathrm{ml}\right)$ was added to the upper chamber and $600 \mu$ l RPMI-1640 medium containing 10\% FBS was added to the lower chamber. After incubation at $37^{\circ} \mathrm{C}$ for $24 \mathrm{~h}$, the cells that had not invaded were removed using cotton swabs. After washing with PBS, the invaded cells were fixed in $4 \%$ paraformaldehyde for $10 \mathrm{~min}$ at room temperature, and stained with $0.1 \%$ crystal violet for $15 \mathrm{~min}$ at room temperature. The number of invaded cells was counted under an optical microscope (magnification, $\mathrm{x} 100$; Olympus Corporation) in five randomly selected fields of view. 
Dual-luciferase reporter assay. Based on the bioinformatics prediction tool PROMO (alggen.lsi.upc.es/cgi-bin/promo_ v3/promo/promoinit.cgi?dirDB=TF_8.3), FOXP3 was identified as a transcription factor of LINC00885. CaSki and HeLa cells were plated in 24 -well plates $\left(4 \times 10^{5}\right.$ cells/well $)$ and co-transfected with various plasmids as indicated in the figures. The pGL3, pGL3-LINC00885 plasmids (Shanghai GenePharma Co., Ltd.) were co-transfected with sh-FOXP3 or sh-NC into cells using Lipofectamine 2000. Cells were collected $24 \mathrm{~h}$ after transfection, and luciferase activity was analyzed using a Dual-Luciferase Reporter assay kit (Promega Corporation). Relative luciferase activity was normalized to Renilla luciferase (control).

Chromatin immunoprecipitation (ChIP). ChIP assay was performed using the EZ-ChIP ${ }^{\mathrm{TM}}$ Chromatin immunoprecipitation kit (cat. no. 17-371; Sigma Aldrich; Merck KGaA) according to the manufacturer's protocol. A total of $1 \times 10^{7}$ cells were cross-linked with $1 \%$ formaldehyde for $10 \mathrm{~min}$ at room temperature. The cell lysates were sonicated using a $10 \mathrm{sec}$ on and $10 \mathrm{sec}$ off mode for 12 cycles on the ice to obtain chromatin fragments, which was then immunoprecipitated at $4^{\circ} \mathrm{C}$ overnight using anti-FOXP3 $(5 \mu \mathrm{g})$ antibody (cat. no. ab20034; Abcam) and normal IgG complexes. Finally, the DNA was obtained via phenol/chloroform extraction and ethanol precipitation, and RT-qPCR was performed as aforementioned.

Western blotting. Total protein was extracted by ice-cold RIPA lysis buffer (Beyotime Institute of Biotechnology). The concentration of protein was detected using a BCA Protein Quantification kit (Beyotime Institute of Biotechnology). A total of $30 \mu \mathrm{g}$ protein was loaded on a $10 \%$ SDS gel, resolved using SDS-PAGE and then transferred to a PVDF membrane (Invitrogen; Thermo Fisher Scientific, Inc.). After blocking with TBS with $0.1 \%$ Tween-20 (TBST; Beijing Solarbio Science $\&$ Technology Co., Ltd.) containing 5\% non-fat milk for $1 \mathrm{~h}$ at room temperature, the membranes were incubated with primary antibodies against FOXP3 (1:1,000; cat. no. ab20034; Abcam), E-cadherin (1:1,000; cat. no. ab40772; Abcam), Vimentin (1:1,000; cat. no. ab92547; Abcam) or GAPDH $(1: 1,000$; cat. no. ab8245; Abcam) overnight at $4^{\circ} \mathrm{C}$. The following day, the membranes were washed using TBST, and incubated with the HRP-conjugated goat anti-rabbit immunoglobulin $\mathrm{G}(\mathrm{IgG}$; 1:20,000; cat. no. ZB-2301; OriGene Technologies, Inc.) or goat anti-mouse IgG (1:20,000; cat. no. ZB-2305; OriGene Technologies, Inc.) secondary antibodies for $1 \mathrm{~h}$ at room temperature. Antigen-antibody complexes were detected using an enhanced chemiluminescence reagent (Cytiva). The relative expression of genes was analyzed using ImageJ (version 5.0; National Institutes of Health) and normalized to GAPDH.

Statistical analysis. Results are presented as the mean \pm standard deviation, and all experiments were performed in triplicate. The differences within groups were assessed using one-way ANOVA followed by Tukey's post hoc test or an independent samples t-test. Analysis was performed using SPSS (version 20; IBM Corp.) and graphed using GraphPad Prism version 8.0 (GraphPad Software, Inc.). $\mathrm{P}<0.05$ was considered to indicate a statistically significant difference.

\section{Results}

LINC00885 expression is upregulated in CC tissues and cell lines. To examine the clinical significance of LINC00885, the expression of LINC00885 in tissue samples from patients was directly extracted from the GEPIA2 database together with the graph, which showed that the expression of LINC00885 in CC tumor tissue samples $(n=306)$ were significantly higher compared with the normal tissue samples $(n=13)$ (Fig. 1A). Additionally, higher expression of LINC00885 was observed in the four CC cell lines (C-33A, SiHa, HeLa and CaSki) compared with the normal cervical epithelial cell line, Ect1/E6E7 (Fig. 1B), suggesting that LINC00885 may be an oncogenic gene in CC.

Knockdown of LINC00885 reduces cell proliferation of CC cells. To examine the role of LINC00885 in CC cell progression, CaSki and HeLa cells were selected for the loss-of-function assays and LINC00885 expression was significantly reduced by sh-LINC00885\#1 and sh-LINC00885\#2 $48 \mathrm{~h}$ after transfection (Fig. 1C). Subsequently, the CCK-8 assay results showed that these two LINC00885 shRNAs significantly reduced the proliferative capacity of CaSki and HeLa cells when compared with the si-NC-transfected cells (Fig. 1D). As presented in Fig. 1E, knockdown of LINC00885 significantly decreased the number of EdU-positive CC cells, which suggested that the knockdown of LINC00885 reduced proliferation. Together, these results suggest that LINC00885 accelerates the malignant proliferation of CC cells.

LINC00885 increases the invasive capacity of CC cells and expression of EMT-related proteins. Transwell invasion assays showed that the knockdown of LINC00885 reduced the invasive capacity of CC cells (Fig. 2A). In order to assess the effect of LINC00885 on EMT in CC cells, the expression of E-cadherin (epithelial marker) and Vimentin (mesenchymal marker) was evaluated using western blotting. There was a significant increase in E-cadherin expression and decrease in Vimentin expression following knockdown of LINC00885, both in CaSki and HeLa cells (Fig. 2B). Collectively, the results showed that LINC00885 increased invasion and EMT in CC cells.

FOXP3 directly regulates LINC00885 in CC cells. Based on the prediction tool PROMO, FOXP3 was found to be a transcription factor of LINC00885 (Fig. 3A). First, FOXP3 expression was assessed in the CC tissues from the GEPIA2 database. Showing a similar pattern to LINC00885, FOXP3 expression was higher in tumor tissues compared with the normal tissues, indicating its potential oncogenic role in $\mathrm{CC}$ cells (Fig. 3B). In addition, FOXP3 expression was higher in the four CC cell lines compared with Ect1/E6E7 cells (Fig. 3C). To examine the biological functions of FOXP3 in CC cells, specific small shRNA was used to knock down FOXP3 expression in two CC cell lines, CaSki and HeLa cells. The transfection efficiency was evaluated $48 \mathrm{~h}$ post-transfection by western blotting (Fig. 3D). The knockdown of FOXP3 expression significantly reduced LINC00885 expression in CaSki and HeLa cells (Fig. 3E). As indicated in Fig. 3F, the knockdown of FOXP3 significantly reduced the luciferase activity in CaSki and HeLa cells co-transfected with pGL3-LINC00885, whereas the knockdown of FOXP3 had no effect on luciferase 
A

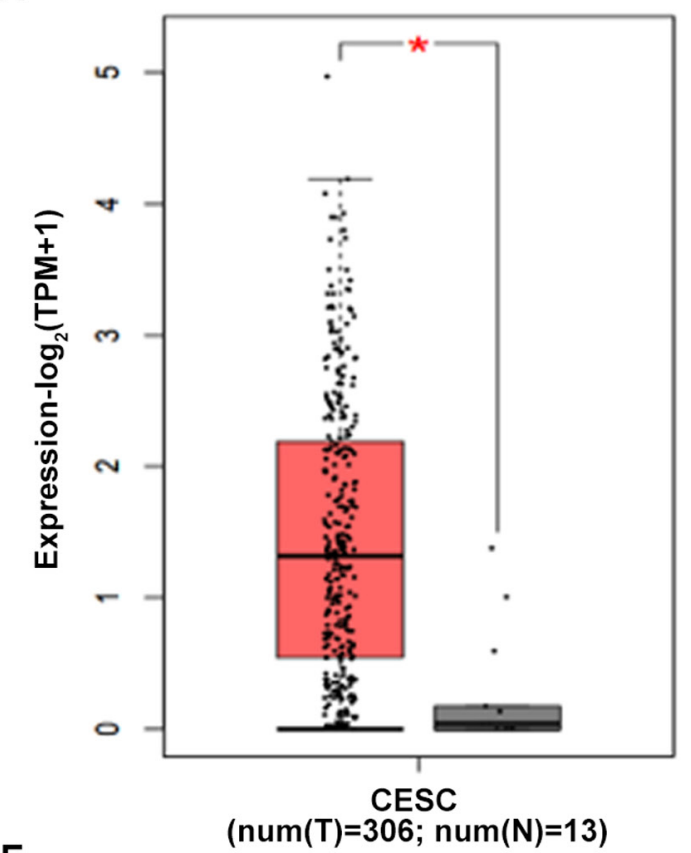

B

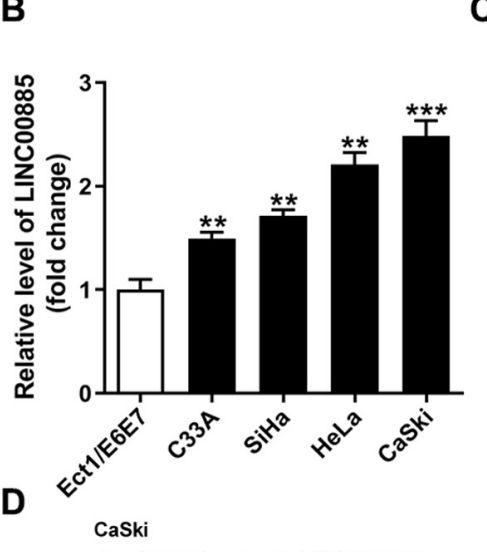

C

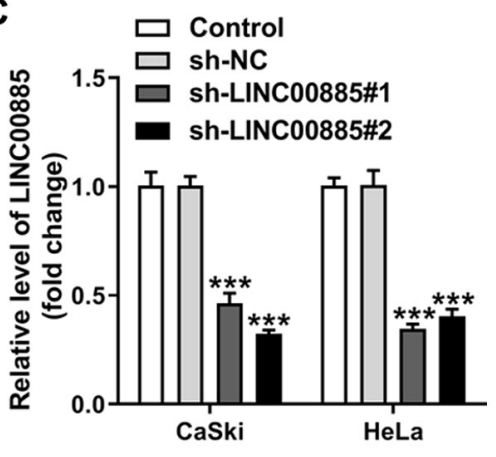

E

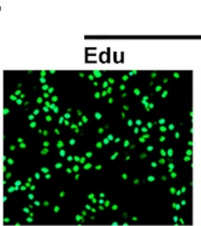

CaSki
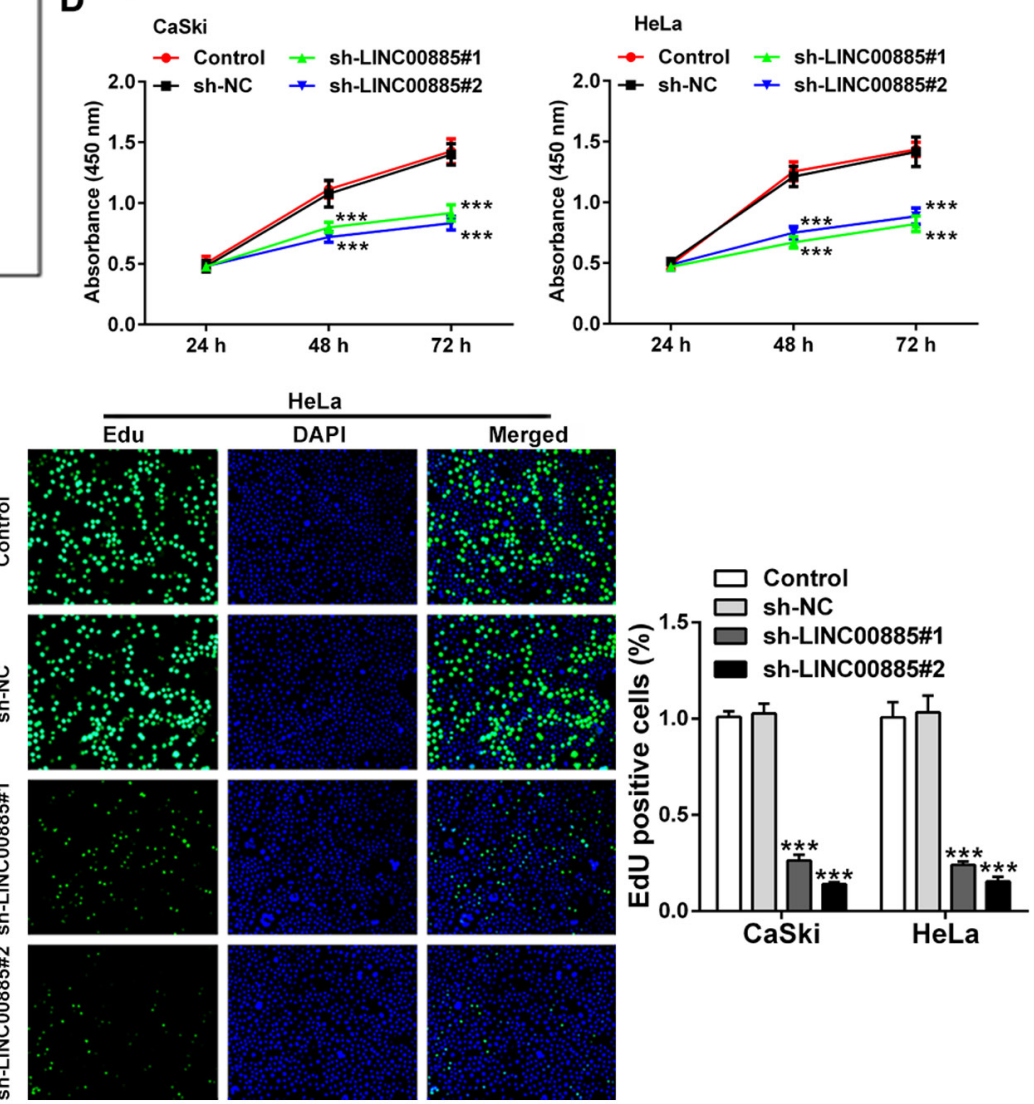

Figure 1. Identification of LINC00885 based on bioinformatics analysis using GEPIA2, and LINC00885 knockdown inhibits the cell proliferation of CC cells. (A) Corresponding box plots of the comparative expression of LINC00885 in CC tumor samples (red) vs. normal tissue samples (grey) generated using GEPIA2. "P<0.01 vs. normal. (B) Relative expression of LINC00885 using GAPDH as the loading control in C-33A, SiHa, HeLa and CaSki CC cell lines, and Ect1/E6E7 normal cervical cells. ${ }^{* *} \mathrm{P}<0.01,{ }^{* * * *} \mathrm{P}<0.001$ vs. Ect1/E6E7. (C) Interference efficiency in CaSki and HeLa cells $48 \mathrm{~h}$ after transfection with two shRNAs against LINC00885 (sh-LINC00885\#1 and sh-LINC00885\#2). (D) Following knockdown of LINC00885, a Cell Counting Kit-8 assay was used to detect changes in the cell proliferation of CaSki and HeLa cells. (E) Knockdown of LINC00885 in CaSki and HeLa cells reduced their proliferative capacities, as shown using an EdU staining assay. ${ }^{* * *} \mathrm{P}<0.001$ vs. control or sh-NC. GEPIA, Gene Expression Profiling Interactive Analysis; CC, cervical cancer; sh, short hairpin RNA; NC, negative control.

activity when the cells were co-transfected with pGL3-NC, suggesting that FOXP3 bound directly to the LINC00885 promoter. ChIP was used to validate the potential endogenous interaction between LINC00885 and FOXP3. The results showed that the LINC00885 promoter was preferentially enriched in the anti-FOXP3 group compared with the IgG control group (Fig. 3G).

Effects of FOXP3 knockdown on the proliferation of CC cells are reversed by LINC00885 overexpression. To verify whether LINC00885 mediated-regulation of the proliferation of CC cells was dependent on FOXP3, LINC00885 was overexpressed in CaSki and HeLa cells using pcDNA-LINC00885 (Fig. 4A). FOXP3 knockdown significantly inhibited the proliferation of CaSki and HeLa cells, and LINC00885 partially reversed such alterations caused by sh-FOXP3 (Fig. 4B). Moreover, as shown in Fig. 4C, the knockdown of FOXP3 significantly reduced the number of EdU-positive (green) CaSki and HeLa cells, and this was restored by pc-LINC00885 transfection. These results showed that FOXP3 directly binds to the promoter of LINC00885, thus indirectly promoting the proliferation of CC cells. 
A
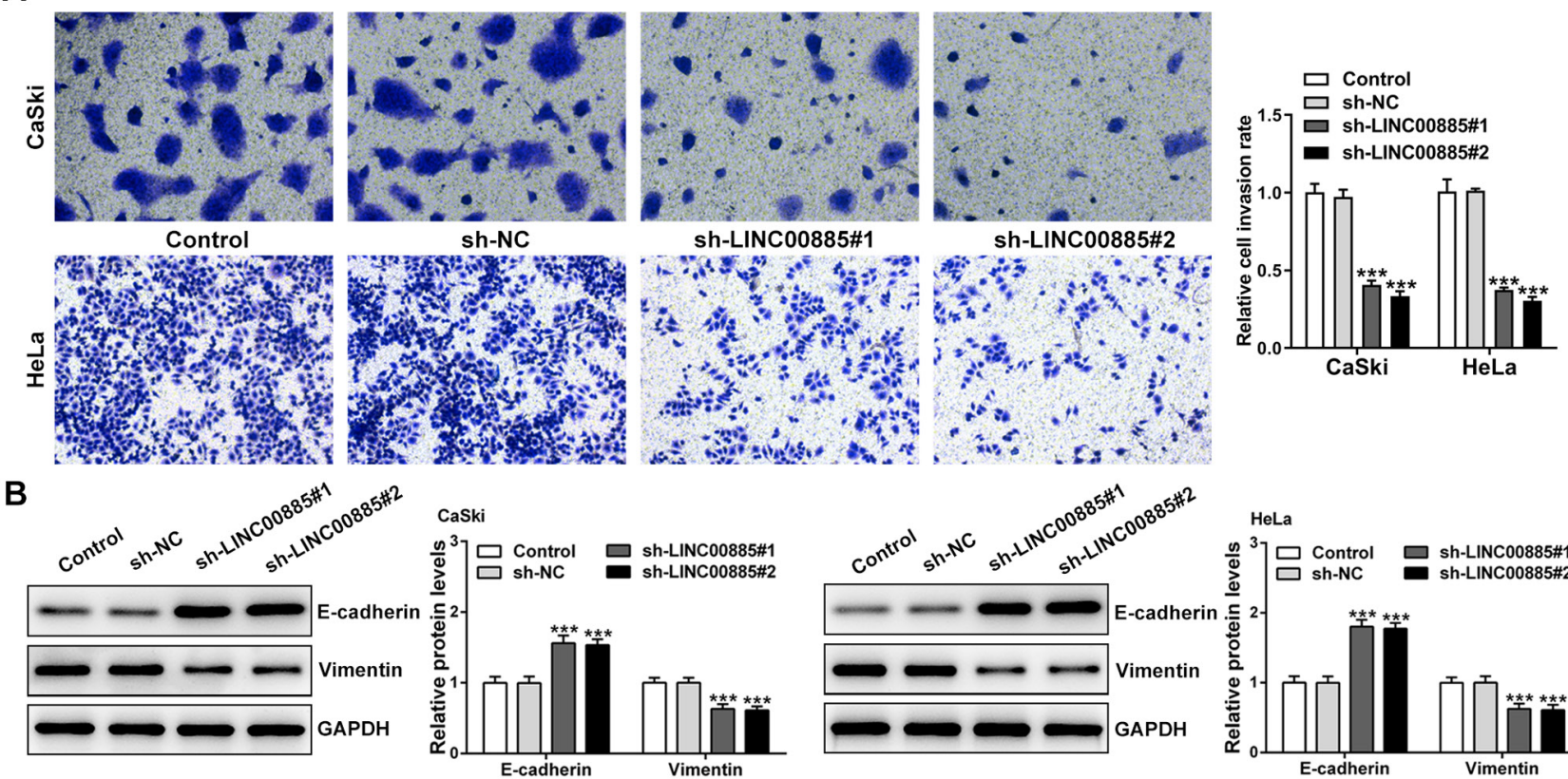

Figure 2. LINC00885 knockdown reduces cell invasion and the expression of EMT-related proteins in cervical cancer cells. (A) Following knockdown of LINC00885, the Transwell assay showed that cell invasion of CaSki and HeLa cells was significantly decreased. Magnification, x200. (B) CaSki and HeLa cells were treated as indicated for $24 \mathrm{~h}$, and total cellular lysates were then collected, subjected to western blot analysis with the indicated antibodies, and densitometry analysis was performed. ${ }^{* * *} \mathrm{P}<0.001$ vs. control or sh-NC. sh, short hairpin RNA; NC, negative control; EMT, epithelial-mesenchymal transition.

Effects of FOXP3 knockdown on the invasion and EMT of CC cells is reversed by LINC00885 overexpression. First, CaSki and HeLa cells were divided into four groups: sh-NC, sh-FOXP3, sh-FOXP3 + pcDNA3.1 and sh-FOXP3 + pc-LINC00885. FOXP3 knockdown alone attenuated the invasive abilities of CaSki and HeLa cells, and the addition of pc-LINC00885 reversed the effects of sh-FOXP3 (Fig. 5A). Likewise, the expression levels of EMT-related factors were also measured by western blotting. As shown in Fig. 5B, the expression levels of E-cadherin were increased, whereas Vimentin expression was decreased after transfection with sh-FOXP3 compared with sh-NC. Overexpression of LINC00885 reduced E-cadherin expression and increased Vimentin expression in both CaSki and HeLa cells. Together, these results demonstrated that LINC00885 leads to EMT of $\mathrm{CC}$ cells via modulation of FOXP3.

\section{Discussion}

$\mathrm{CC}$ is one of the most common types of cancer in women worldwide with high morbidity and mortality rates, and continues to be a serious public health problem in the developing world, including in China (26). Due to its large population and the geographical and socioeconomic inequities, China has a high burden of CC and considerable disparities in the capacity to detect and manage the disease amongst different regions (26). For patients with $\mathrm{CC}$, there are two types of metastasis: Hematogenous metastasis and lymphatic metastasis. Patients with hematogenous metastasis have a higher risk of death compared with those with lymphatic metastasis, and the metastatic sites include the lungs, liver, bone and brain (27). Thus, the identification of novel suitable prognostic biomarkers is critical to improve the detection of CC.
LINC00885 is a newly discovered lncRNA with few relevant studies, and its abnormal expression has been observed only in human breast and bladder cancer $(10,11)$. To the best of our knowledge, the present study was the first to show the increased expression of LINC00885 in CC and its crucial role in the acquisition of a malignant phenotype in CC cells. In the present study, based on the analysis of data obtained from TCGA and GTEx, the expression of LINC00885 in CC tissues was notably higher compared with the normal cervical tissues. Additionally, the expression of LINC00885 in CC cells was consistent with TCGA and GTEx data. These findings suggested that LINC00885 may act as a carcinogenic lncRNA involved in the pathophysiology of CC. In order to further identify whether LINC00885 contributes to the proliferation, invasion and EMT of CC, loss-of-function experiments were performed. The proliferative and invasive abilities, and extent of EMT of CC CaSki and HeLa cells were notably decreased in cells transfected with two different LINC00885 shRNAs, which showed that LINC00885 served a crucial role in maintaining the malignant phenotype of CC. Studies on other lncRNAs have found similar results in CC, such as HOTAIR (28), MALAT1 (29) and EBIC (30). In brief, overexpression of HOTAIR can be used as a predictor for poor prognosis of CC and suppression of IncRNA HOTAIR can reduce the proliferation, migration and invasion of $\mathrm{CC}$ cells and decrease the expression of EMT-related proteins (28). Downregulation of IncRNA-MALATl expression can reduce the proliferative and invasive capacities of CC cells and was correlated with HPV in cervical cancer (29). Furthermore, the lncRNA-EBIC-mediated increase in CC cell infiltration may be related to inhibition of E-cadherin expression (30).

FOXP3 is a specific biomarker commonly used to identify Tregs; Tregs suppress the antitumor immune response, and 
A

\begin{tabular}{|ll|}
\hline Transcription factors of LINC00885: \\
YY1 & HNF-3 $\alpha$ \\
GR & RXR- $\alpha$ \\
C/EBP $\beta$ & TFIID \\
GR- $\alpha$ & C-Myc \\
ER- $\alpha$ & STAT4 \\
SRY & TFII-I \\
FOXP3 & GR- $\beta$ \\
HNF-1A & Pax-5 \\
NF-1 & IRF-2 \\
\hline
\end{tabular}

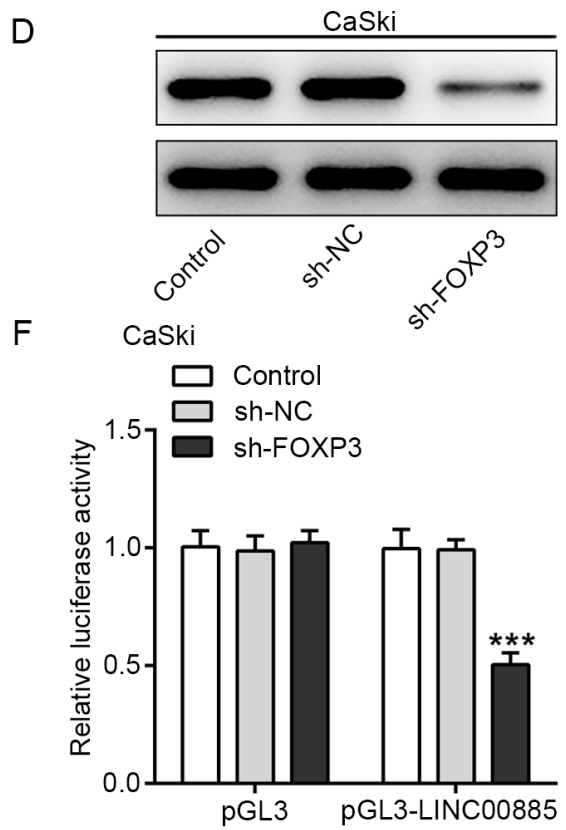

B

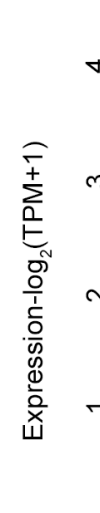

C

G

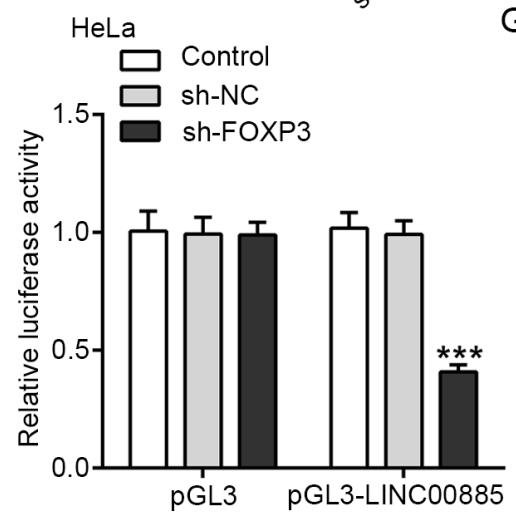

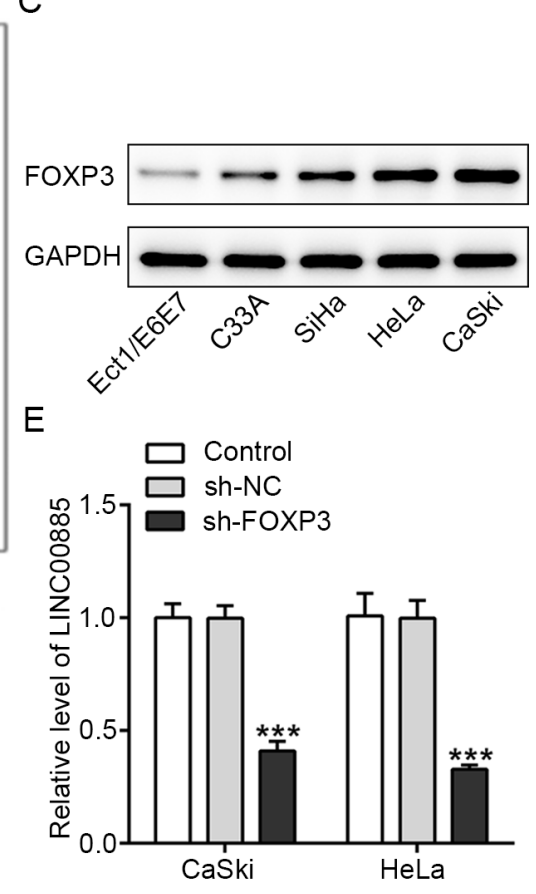

$\mathrm{E}$
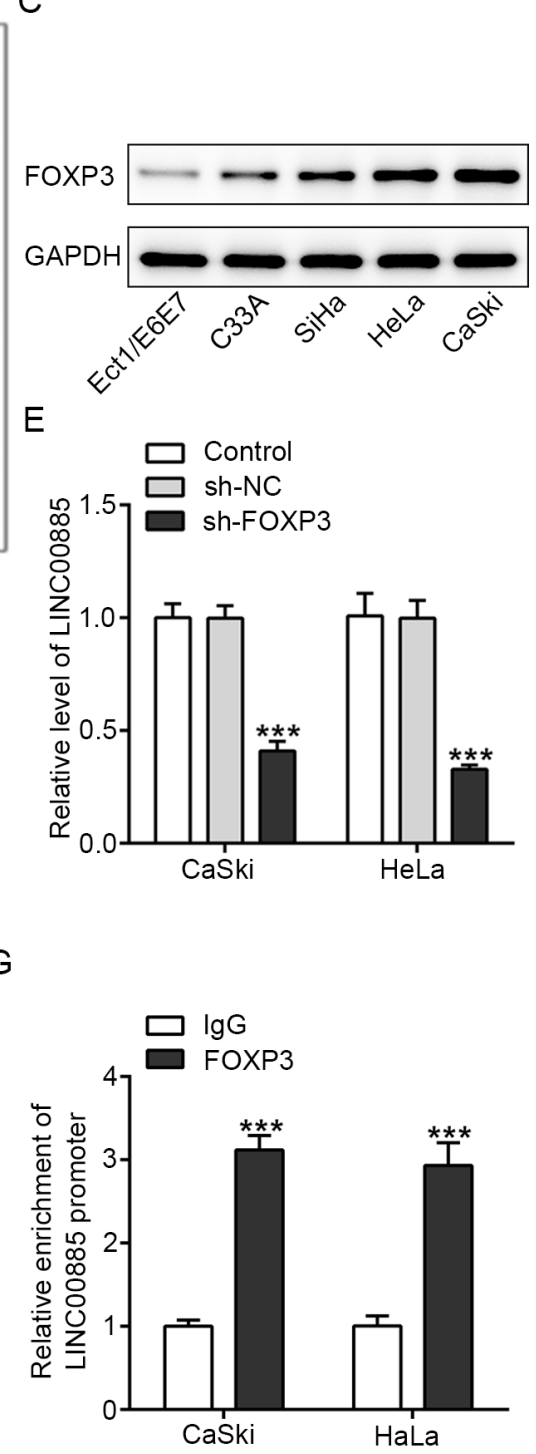

Figure 3. Regulation of screening and verification of FOXP3 by LINC00885 in CC. (A) Bioinformatics prediction tool PROMO showed the transcription factors of LINC00885. (B) Corresponding box plots of the comparative expression of FOXP3 in CC tumor samples (red) vs. normal tissue samples (grey) were generated using Gene Expression Profiling Interactive Analysis 2. ${ }^{*} \mathrm{P}<0.05$ vs. normal. (C) Expression of FOXP3 was elevated in four CC cell lines based on western blot analysis. (D) Western blotting was used to assess the knockdown efficiency of sh-FOXP3 in CaSki and HeLa cells. (E) After knockdown of FOXP3 in CaSki and HeLa cells, LINC00885 expression was significantly reduced. (F) The luciferase reporter assay showed that the knockdown of FOXP3 significantly reduced the luciferase activity in cells that were transfected with the pGL3-LINC00885 vector without reducing the luciferase activity in cells that were transfected with the pGL3-NC vector. ${ }^{* * *} \mathrm{P}<0.001$ vs. control or sh-NC. (G) Chromatin immunoprecipitation experiments were performed in CaSki and HeLa cells, and the co-precipitated FOXP3 was subjected to reverse transcription-quantitative PCR to assess LINC00885 promoter expression. ${ }^{* * * *} \mathrm{P}<0.001$ vs. IgG. CC, cervical cancer; sh, short hairpin RNA; NC, negative control; FOXP3, forkhead box protein 3.

thereby promote tumor progression. The roles of FOXP3 in cervical cancer has been previously demonstrated $(20,21)$. Thus, it was not surprising that FOXP3 participated in the acquisition of a malignant phenotype in CC cells, including proliferation, invasion and EMT, which can be suppressed by the knockdown of FOXP3. Following the knockdown of FOXP3, the ectopic overexpression of LINC00885 partially restored the malignant phenotype of $\mathrm{CC}$ cells, which also highlighted a potential association between LINC00885 and FOXP3. Furthermore, FOXP3 is a transcription factor of LINC00885, and this was verified in the present study based on the results of the dual-luciferase reporter and ChIP assays. Therefore, it was hypothesized that LINC00885 promotes CC development via regulation of FOXP3 expression. Although further investigation is required, the results of the present study showed that LINC00885 and FOXP3 may function as a tumor promoter in CC.

In conclusion, FOXP3 regulated the effects of LINC00885 on the proliferation, invasion and EMT of CC cells. LINC00885 and FOXP3 may thus serve as biomarkers for the early diagnosis of $\mathrm{CC}$ and a potential therapeutic target for reversing the malignant phenotype of CC. Finally, the combined results provided a novel perspective of FOXP3 and LINC00885 function in papillary CC tumorigenesis.

\section{Acknowledgements}

Not applicable. 
A

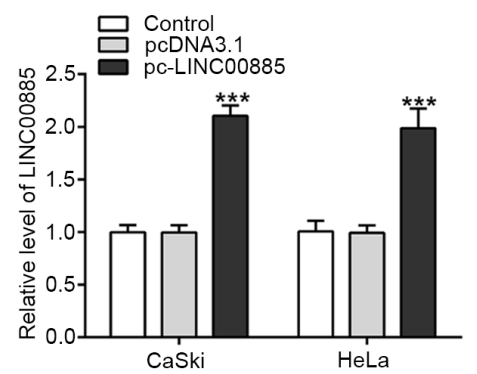

C
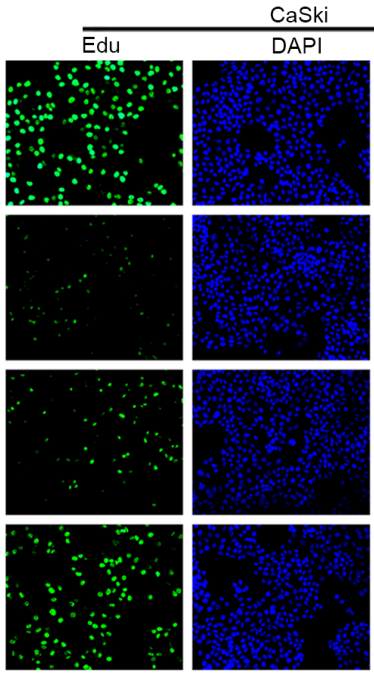

B
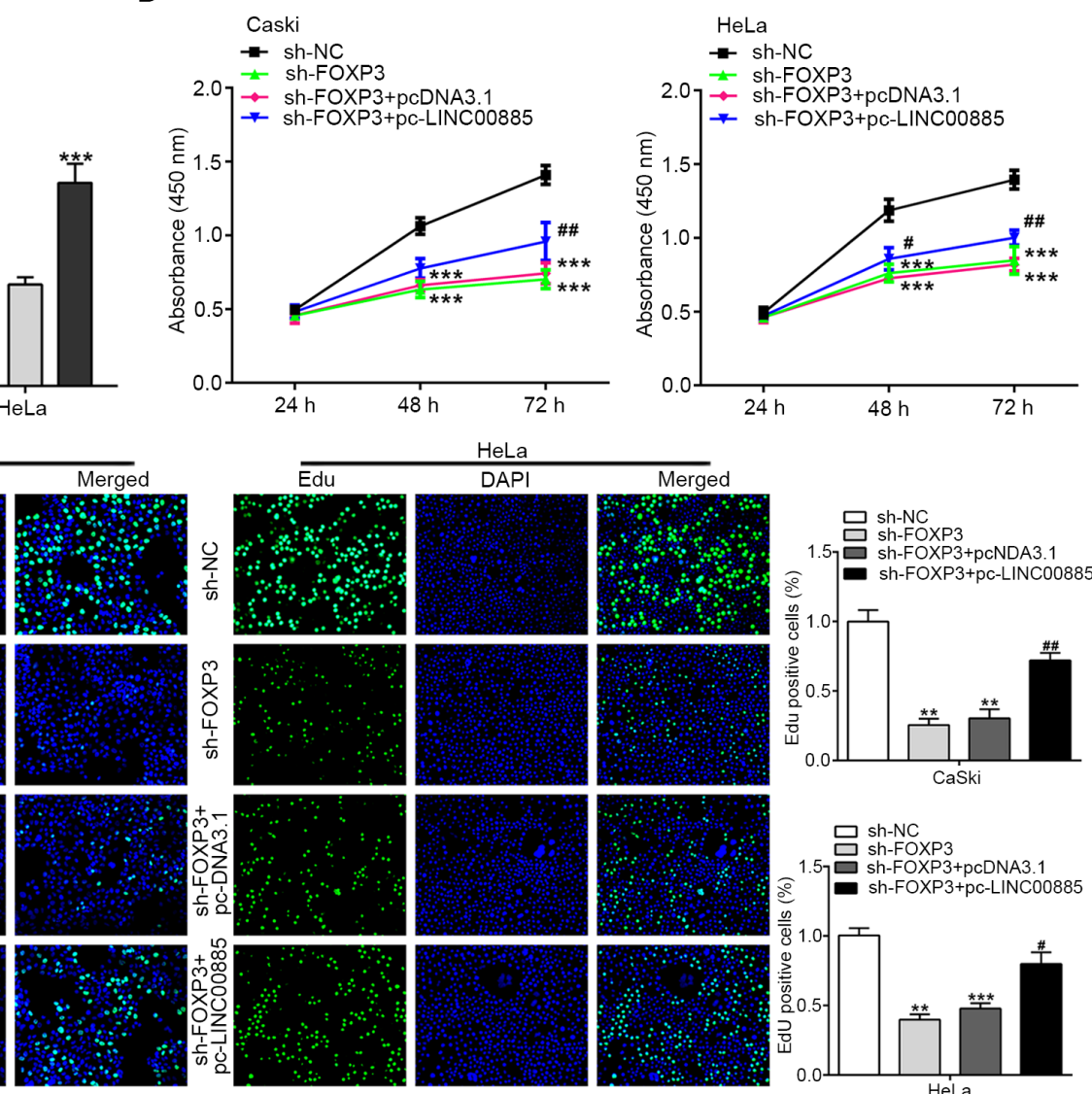

Figure 4. Inhibitory effect of FOXP3 knockdown on the proliferation of CaSki and HeLa cells are reversed by overexpression of LINC00885. (A) Expression of LINC00885 was significantly increased in CaSki and HeLa cells following transfection with pc-LINC00885. ${ }^{* * *} \mathrm{P}<0.001$ vs. control and pcDNA3.1. CaSki and HeLa cells were treated as indicated, and cell proliferation was examined using (B) MTT and (C) EdU staining assays. ${ }^{* * *} \mathrm{P}<0.01,{ }^{* * *} \mathrm{P}<0.001 \mathrm{vs}$. sh-NC; ${ }^{*} \mathrm{P}<0.05$, ${ }^{\#} \mathrm{P}<0.01$ vs. sh-FOXP3 or sh-FOXP3 + pcDNA3.1. FOXP3, forkhead box protein 3; sh, short hairpin RNA; NC, negative control.

A
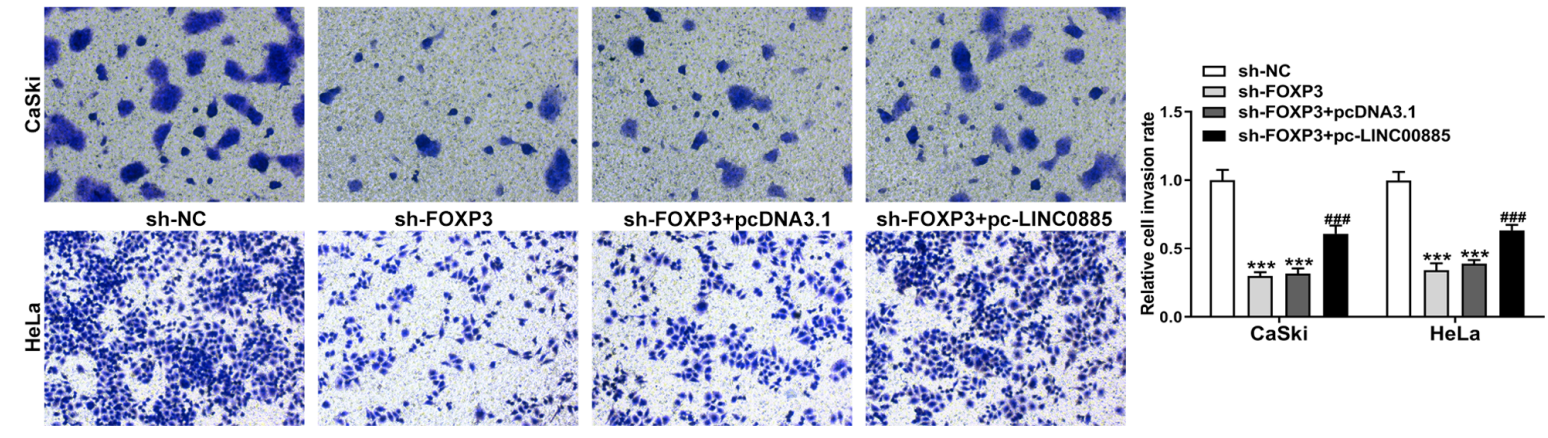

B
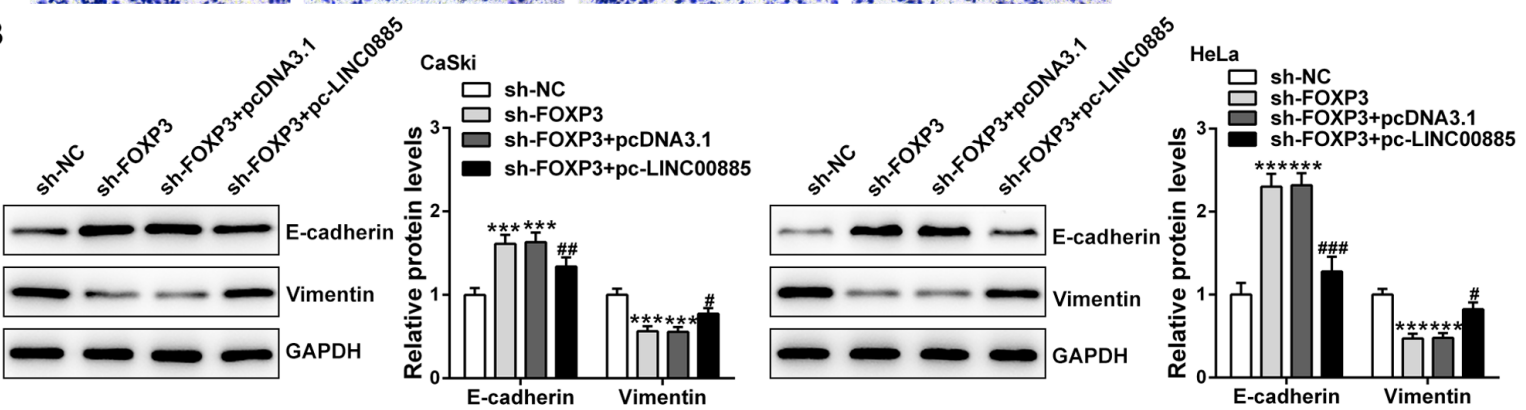

Figure 5. Inhibitory effect of FOXP3 knockdown on the invasion and EMT of CaSki and HeLa cells is reversed by LINC00885 overexpression. (A) Invasion assay of CaSki and HeLa cells treated as indicated. After $48 \mathrm{~h}$, the number of cells that had invaded through the membrane were counted under a microscope in five random fields. Magnification, x200. (B) Expression of EMT-related proteins E-cadherin and Vimentin in CaSki and HeLa cells treated as indicated were detected using western blotting. ${ }^{* * *} \mathrm{P}<0.001$ vs. sh-NC; ${ }^{\#} \mathrm{P}<0.05,{ }^{\# \#} \mathrm{P}<0.01,{ }^{\# \#} \mathrm{P}<0.001$ vs. sh-FOXP3 and sh-FOXP3 + pcDNA3.1. EMT, epithelial-mesenchymal transition; sh, short hairpin RNA; FOXP3, forkhead box protein 3; NC, negative control. 


\section{Funding}

No funding was received.

\section{Availability of data and materials}

All data generated or analyzed during this study are included in this published article.

\section{Authors' contributions}

JX and LL conceived and designed the study. YL, HT and LZ performed the experiments. YL and HT analyzed the data. YL and LL wrote the manuscript. JX revised the manuscript. All authors read and approved the final manuscript. YL, JX and LL are responsible for confirming the authenticity of the raw data.

\section{Ethics approval and consent to participate}

Not applicable.

\section{Patient consent for publication}

Not applicable.

\section{Competing interests}

The authors declare that they have no competing interests.

\section{References}

1. Bray F, Ferlay J, Soerjomataram I, Siegel RL, Torre LA and Jemal A: Global cancer statistics 2018: GLOBOCAN estimates of incidence and mortality worldwide for 36 cancers in 185 countries. CA Cancer J Clin 68: 394-424, 2018

2. Shrestha AD, Neupane D, Vedsted P and Kallestrup P: Cervical cancer prevalence, incidence and mortality in low and middle income countries: A systematic review. Asian Pac J Cancer Prev 19: 319-324, 2018.

3. Ferlay J, Steliarova-Foucher E, Lortet-Tieulent J, Rosso S, Coebergh JW, Comber H, Forman D and Bray F: Cancer incidence and mortality patterns in Europe: Estimates for 40 countries in 2012. Eur J Cancer 49: 1374-1403, 2013.

4. Canfell K: Towards the global elimination of cervical cancer. Papillomavirus Res 8: 100170, 2019.

5. Gupta RA, Shah N, Wang KC, Kim J, Horlings HM, Wong DJ, Tsai MC, Hung T, Argani P, Rinn JL, et al: Long non-coding RNA HOTAIR reprograms chromatin state to promote cancer metastasis. Nature 464: 1071-1076, 2010.

6. Tano $\mathrm{K}$ and Akimitsu N: Long non-coding RNAs in cancer progression. Front Genet 3: 219, 2012.

7. Hu Y, Chen HY, Yu CY, Xu J, Wang JL, Qian J, Zhang X and Fang JY: A long non-coding RNA signature to improve prognosis prediction of colorectal cancer. Oncotarget 5: 2230-2242, 2014.

8. Zhu X, Tian X, Yu C, Shen C, Yan T, Hong J, Wang Z, Fang JY and Chen $\mathrm{H}$ : A long non-coding RNA signature to improve prognosis prediction of gastric cancer. Mol Cancer 15: 60, 2016.

9. Li J, Wang W, Xia P, Wan L, Zhang L, Yu L, Wang L, Chen X, $\mathrm{Xiao} \mathrm{Y}$ and $\mathrm{Xu} \mathrm{C}$ : Identification of a five-lncRNA signature for predicting the risk of tumor recurrence in patients with breast cancer. Int J Cancer 143: 2150-2160, 2018.

10. Zaheed O, Samson J and Dean K: A bioinformatics approach to identify novel long, non-coding RNAs in breast cancer cell lines from an existing RNA-sequencing dataset. Noncoding RNA Res 5: 48-59, 2020.
11. Li M, Liu Y, Zhang X, Liu J and Wang P: Transcriptomic analysis of high-throughput sequencing about circRNA, lncRNA and mRNA in bladder cancer. Gene 677: 189-197, 2018.

12. Huang Y, Liao H, Zhang Y, Yuan R, Wang F, Gao Y, Wang P and Du Z: Prognostic value of tumor-infiltrating FoxP3+ T cells in gastrointestinal cancers: A meta analysis. PLoS One 9: e94376, 2014.

13. Zhang Y, Huang Y and Qin M: Tumour-infiltrating FoxP3+ and IL-17-producing $\mathrm{T}$ cells affect the progression and prognosis of gallbladder carcinoma after surgery. Scand J Immunol 78: 516-522, 2013

14. Bennett CL, Christie J, Ramsdell F, Brunkow ME, Ferguson PJ, Whitesell L, Kelly TE, Saulsbury FT, Chance PF and Ochs HD: The immune dysregulation, polyendocrinopathy, enteropathy, $\mathrm{X}$-linked syndrome (IPEX) is caused by mutations of FOXP3. Nat Genet 27: 20-21, 2001.

15. Peng GL, Li L, Guo YW, Yu P, Yin XJ, Wang S and Liu CP: CD8(+) cytotoxic and FoxP3(+) regulatory T lymphocytes serve as prognostic factors in breast cancer. Am J Transl Res 11: 5039-5053, 2019.

16. Li F, Sun Y, Huang J, Xu W, Liu J and Yuan Z: CD4/CD8 + $\mathrm{T}$ cells, DC subsets, Foxp3, and IDO expression are predictive indictors of gastric cancer prognosis. Cancer Med 8: 7330-7344, 2019.

17. Wang S, Wu J, Ren J, Vlantis AC, Li MY, Liu SYW, Ng EKW, Chan ABW, Luo DC, Liu Z, et al: MicroRNA-125b interacts with Foxp3 to induce autophagy in thyroid cancer. Mol Ther 26: 2295-2303, 2018.

18. Yang S, Liu Y, Li MY, Ng CSH, Yang SL, Wang S, Zou C, Dong Y, Du J, Long X, et al: FOXP3 promotes tumor growth and metastasis by activating Wnt/beta-catenin signaling pathway and EMT in non-small cell lung cancer. Mol Cancer 16: 124, 2017.

19. Tanaka A and Sakaguchi S: Targeting Treg cells in cancer immunotherapy. Eur J Immunol 49: 1140-1146, 2019.

20. Luo Q, Zhang S, Wei H, Pang X and Zhang H: Roles of Foxp3 in the occurrence and development of cervical cancer. Int J Clin Exp Pathol 8: 8717-8730, 2015.

21. Tang J, Yang Z, Wang Z, Li Z, Li H, Yin J, Deng M, Zhu W and Zeng C: Foxp3 is correlated with VEGF-C expression and lymphangiogenesis in cervical cancer. World J Surg Oncol 15: $173,2017$.

22. Tang Z, Kang B, Li C, Chen T and Zhang Z: GEPIA2: An enhanced web server for large-scale expression profiling and interactive analysis. Nucleic Acids Res 47: W556-W560, 2019.

23. Wang Z, Jensen MA and Zenklusen JC: A practical guide to The Cancer Genome Atlas (TCGA). Methods Mol Biol 1418: 111-141, 2016.

24. GTEx Consortium: The Genotype-Tissue Expression (GTEx) project. Nat Genet 45: 580-585, 2013.

25. Livak KJ and Schmittgen TD: Analysis of relative gene expression data using real-time quantitative PCR and the 2(-Delta Delta C(T)) method. Methods 25: 402-408, 2001.

26. Di J, Rutherford S and Chu C: Review of the cervical cancer burden and population-based cervical cancer screening in China. Asian Pac J Cancer Prev 16: 7401-7407, 2015.

27. $\mathrm{Li} \mathrm{H}, \mathrm{Wu} \mathrm{X}$ and Cheng $\mathrm{X}$ : Advances in diagnosis and treatment of metastatic cervical cancer. J Gynecol Oncol 27: e43, 2016.

28. Kim HJ, Lee DW, Yim GW, Nam EJ, Kim S, Kim SW and Kim YT: Long non-coding RNA HOTAIR is associated with human cervical cancer progression. Int J Oncol 46: 521-530, 2015.

29. Jiang Y, Li Y, Fang S, Jiang B, Qin C, Xie P, Zhou G and Li G: The role of MALAT1 correlates with HPV in cervical cancer. Oncol Lett 7: 2135-2141, 2014.

30. Sun NX, Ye C, Zhao Q, Zhang Q, Xu C, Wang SB, Jin ZJ, Sun SH, Wang $\mathrm{F}$ and $\mathrm{Li} \mathrm{W}$ : Long noncoding RNA-EBIC promotes tumor cell invasion by binding to EZH2 and repressing E-cadherin in cervical cancer. PLoS One 9: e100340, 2014.

This work is licensed under a Creative Commons Attribution-NonCommercial-NoDerivatives 4.0 International (CC BY-NC-ND 4.0) License. 